\title{
Clear correlation between monopoles and the chiral condensate in $\mathrm{SU}(3) \mathrm{QCD}$
}

\author{
Hiroki Ohata ${ }^{\circ}$ \\ Yukawa Institute for Theoretical Physics, Kyoto University, Kyoto 606-8502, Japan \\ Hideo Suganuma $\odot$ \\ Department of Physics, Kyoto University, Kitashirakawaoiwake, Sakyo, Kyoto 606-8502, Japan
}

(Received 11 December 2020; revised 18 February 2021; accepted 23 February 2021; published 19 March 2021)

\begin{abstract}
We study spontaneous chiral-symmetry breaking in SU(3) QCD in terms of the dual superconductor picture for quark confinement in the maximally Abelian (MA) gauge, using lattice QCD Monte Carlo simulations with four different lattices of $16^{4}, 24^{4}, 24^{3} \times 6$ at $\beta=6.0$ (i.e., the spacing $a \simeq 0.1 \mathrm{fm}$ ), and $32^{4}$ at $\beta=6.2$ (i.e., $a \simeq 0.075 \mathrm{fm}$ ), at the quenched level. First, in the confinement phase, we find Abelian dominance and monopole dominance in the MA gauge for the chiral condensate in the chiral limit, using the two different methods of (i) the Banks-Casher relation with the Dirac eigenvalue density and (ii) finite quark-mass calculations with the quark propagator and its chiral extrapolation. In the hightemperature deconfined phase, the chiral restoration is observed also for the Abelian and the monopole sectors. Second, we investigate local correlation between the chiral condensate and monopoles, which topologically appear in the MA gauge. We find that the chiral condensate locally takes a quite large value near monopoles. As an interesting possibility, the strong magnetic field around monopoles is responsible to chiral symmetry breaking in QCD, similarly to the magnetic catalysis.
\end{abstract}

DOI: 10.1103/PhysRevD.103.054505

\section{INTRODUCTION}

Since quantum chromodynamics (QCD) was established as the fundamental theory of strong interaction in 1970s, to understand its nonperturbative properties has been one of the most difficult central problems in theoretical physics for about a half century. In particular, QCD exhibits two outstanding nonperturbative phenomena of quark confinement and spontaneous chiral-symmetry breaking in its lowenergy region, many physicists have tried to clarify these phenomena and their relation directly from QCD, but this is still an unsolved important issue in the particle physics.

Chiral symmetry breaking in QCD is categorized as wellknown spontaneous symmetry breaking, which widely appears in various fields in physics, and is an important phenomenon relating to dynamical quark-mass generation $[1,2]$. Apart from the dark matter, about $99 \%$ of the matter mass of our Universe originates from chiral symmetry breaking, because the Higgs-origin mass is just a small mass of $u, d$ current quarks, electrons, and neutrinos [3]. The order parameter of chiral symmetry breaking is the

Published by the American Physical Society under the terms of the Creative Commons Attribution 4.0 International license. Further distribution of this work must maintain attribution to the author(s) and the published article's title, journal citation, and DOI. Funded by SCOAP ${ }^{3}$. chiral condensate $\langle\bar{q} q\rangle$, and it is directly related to low-lying Dirac modes via the Banks-Casher relation [4].

In contrast, color confinement is a fairly unique phenomenon peculiar in QCD, and quark confinement is characterized by the linear interquark potential. As for the confinement mechanism, the dual superconductor picture based on color-magnetic monopole condensation was proposed by Nambu, 't Hooft, and Mandelstam as a typical plausible physical scenario [5-7]. In lattice QCD, by taking the maximally Abelian (MA) gauge [8], this dual superconductor scenario has been investigated in terms of Abelian dominance, i.e., dominant role of the Abelian sector [9-12]. and the relevant role of monopoles [13-15].

The relation between confinement and chiral symmetry breaking is not yet clarified directly from QCD. While a strong correlation between confinement and chiral symmetry breaking has been suggested by almost coincidence between deconfinement and chiral-restoration temperatures [16], an lattice QCD analysis based on the Dirac-mode expansion indicates some independence of these phenomena [17].

Their correlation has been also suggested in terms of color-magnetic monopoles, which topologically appear in QCD in the Abelian gauge [18]. In the dual GinzburgLandau theory, the monopole condensate is responsible to chiral symmetry breaking as well as quark confinement [19]. Also in SU(2) lattice QCD, Miyamura and Woloshyn 
showed Abelian dominance [20,21] and monopole dominance $[20,22]$ for chiral symmetry breaking. In fact, by removing the monopoles from the QCD vacuum, confinement and chiral symmetry breaking are simultaneously lost. In SU(3) lattice QCD with a $8^{3} \times 4$ lattice, Thurner et al. showed a local correlation among monopoles, instantons, and the chiral condensate [23]. These studies indicate an important role of the monopoles to both phenomena, and thus these two phenomena might be related via the monopole. However, most of the pioneering lattice works were done in SU(2) lattice QCD or done on a small lattice [20-23].

In this paper, we investigate correlation between chiral symmetry breaking and color-magnetic monopoles appearing in the MA gauge in SU(3) lattice QCD with large-volume fine lattices at the quenched level. Using two different methods, we evaluate the chiral condensate in Abelianized QCD and the monopole system, extracted from lattice QCD. We also investigate correlation between the local chiral-condensate value and the monopole location.

\section{LATTICE SETUP AND ABELIAN PROJECTION}

We perform SU(3) lattice QCD simulations at the quenched level with the standard plaquette action [16]. On four-dimensional Euclidean lattices, the gauge variable is described as the $\mathrm{SU}(3)$ link variable $U_{\mu}(s) \equiv e^{i a g A_{\mu}(s)} \in$ $\mathrm{SU}(3)$, with the gluon field $A_{\mu}(s) \in \mathrm{su}(3)$, the QCD gauge coupling $g$, and the lattice spacing $a$. The lattice spacing $a$ is determined so as to reproduce the string tension $\sigma=0.89 \mathrm{GeV} / \mathrm{fm}[11]$.

In this work, we use four different lattices with the size and the lattice parameter $\beta \equiv 6 / g^{2}$ :

(a) $16^{3} \times 16$ and $\beta=6.0$ (i.e., $a \simeq 0.1 \mathrm{fm}$ ),

(b) $24^{3} \times 24$ and $\beta=6.0$,

(c) $32^{3} \times 32$ and $\beta=6.2$ (i.e., $a \simeq 0.075 \mathrm{fm}$ ),

(d) $24^{3} \times 6$ and $\beta=6.0$.

From the first and the second lattices, the finite volume effect can be checked. Since the second and the third lattices have almost the same physical volume, the finite lattice-spacing effect can be also checked. The last one exhibits the high-temperature deconfined phase at $T \simeq$ $330 \mathrm{MeV}$ above the critical temperature. In each direction, the periodic boundary condition is imposed for link variables, and the antiperiodic boundary condition for quarks, which realizes the finite temperature system for the last lattice.

Hereafter, we take the lattice unit $a=1$. Using the pseudo-heat-bath algorithm, we generate 300, 100, 100, and 300 gauge configurations for the lattices of (a), (b), (c), and (d), respectively. All of gauge configurations are taken every 500 sweeps after a thermalization of 5000 sweeps. We use the jackknife method for the statistical error estimate.
Using the Cartan subalgebra $\vec{H} \equiv\left(T_{3}, T_{8}\right)$ of $\mathrm{SU}(3)$, the MA gauge fixing is defined so as to maximize

$$
\begin{aligned}
R_{\mathrm{MA}}\left[U_{\mu}(s)\right] & \equiv \sum_{s} \sum_{\mu=1}^{4} \operatorname{tr}\left(U_{\mu}^{\dagger}(s) \vec{H} U_{\mu}(s) \vec{H}\right), \\
& =\sum_{s} \sum_{\mu=1}^{4}\left(1-\frac{1}{2} \sum_{i \neq j}\left|U_{\mu}(s)_{i j}\right|^{2}\right),
\end{aligned}
$$

under the $\mathrm{SU}(3)$ gauge transformation, and thus this gauge fixing suppresses all the off-diagonal fluctuation of the $\mathrm{SU}(3)$ field $U_{\mu}(s)$. In the MA gauge, the $\mathrm{SU}(3)$ gauge group is partially fixed remaining its maximal torus subgroup $\mathrm{U}(1)_{3} \times \mathrm{U}(1)_{8}$, and $\mathrm{QCD}$ is reduced into an Abelian gauge theory like the non-Abelian Higgs theory.

In this work, the MA gauge fixing is performed with the stopping criterion that the deviation $\Delta R_{\mathrm{MA}} /\left(4 V^{4}\right)$ becomes smaller than $10^{-5}$ in 100 iterations.

From the $\mathrm{SU}(3)$ field $U_{\mu}^{\mathrm{MA}}(s) \in \mathrm{SU}(3)$ in the MA gauge, the Abelian field is defined as

$u_{\mu}(s)=e^{i \vec{\theta} \cdot \vec{H}}=\operatorname{diag}\left(e^{i \theta_{\mu}^{1}(s)}, e^{i \theta_{\mu}^{2}(s)}, e^{i \theta_{\mu}^{3}(s)}\right) \in \mathrm{U}(1)^{2}$,

with the constraint $\sum_{i=1}^{3} \theta_{\mu}^{i}(s)=0(\bmod 2 \pi)$, by maximizing the overlap

$$
R_{\text {Abel }} \equiv \frac{1}{3} \operatorname{Re} \operatorname{tr}\left\{U_{\mu}^{\mathrm{MA}}(s) u_{\mu}^{\dagger}(s)\right\} \in\left[-\frac{1}{2}, 1\right],
$$

so that the distance between $u_{\mu}(s)$ and $U_{\mu}^{\mathrm{MA}}(s)$ becomes the smallest in the SU(3) manifold.

The Abelian projection is defined by the replacement of $\mathrm{SU}(3)$ fields $U_{\mu}(s)$ by Abelian fields $u_{\mu}(s)$ for each gauge configuration, i.e., $O\left[U_{\mu}(s)\right] \rightarrow O\left[u_{\mu}(s)\right]$ for QCD operators. In this way, Abelian-projected QCD is extracted from $\mathrm{SU}(3) \mathrm{QCD}$. The case of $\left\langle O\left[U_{\mu}(s)\right]\right\rangle \simeq\left\langle O\left[u_{\mu}(s)\right]\right\rangle$ is called "Abelian dominance" for the operator $O$.

\section{MONOPOLES IN QCD}

Now, let us consider the Abelian plaquette variable,

$$
\begin{aligned}
u_{\mu \nu}(s) & \equiv u_{\mu}(s) u_{\nu}(s+\hat{\mu}) u_{\mu}^{\dagger}(s+\hat{\nu}) u_{\nu}^{\dagger}(s)=e^{i \theta_{\mu \nu}(s)}, \\
& =\operatorname{diag}\left(e^{i \theta_{\mu \nu}^{1}(s)}, e^{i \theta_{\mu \nu}^{2}(s)}, e^{i \theta_{\mu \nu}^{3}(s)}\right) \in \mathrm{U}(1)^{2},
\end{aligned}
$$

where $\hat{\mu}$ is the $\mu$-directed unit vector in the lattice unit. The Abelian field strength $\theta_{\mu \nu}^{i}(s)(i=1,2,3)$ is the principal value of the exponent in $u_{\mu \nu}(s)$, and is defined as

$$
\begin{gathered}
\partial_{\mu} \theta_{\nu}^{i}(s)-\partial_{\nu} \theta_{\mu}^{i}(s)=\theta_{\mu \nu}^{i}(s)-2 \pi n_{\mu \nu}^{i}(s), \\
-\pi \leq \theta_{\mu \nu}^{i}(s)<\pi, \quad n_{\mu \nu}^{i}(s) \in \mathbb{Z},
\end{gathered}
$$

with the forward derivative $\partial_{\mu}$. Here, $\theta_{\mu \nu}^{i}(s)$ is $\mathrm{U}(1)^{2}$ gauge invariant and corresponds to the regular continuum Abelian 
field strength as $a \rightarrow 0$, while $n_{\mu \nu}^{i}(s)$ corresponds to the singular gauge-variant Dirac string [24].

The electric current $j_{\mu}^{i}$ and the monopole current $k_{\mu}^{i}$ are defined from the Abelian field strength $\theta_{\mu \nu}^{i}$,

$$
\begin{gathered}
j_{\nu}^{i}(s) \equiv \partial_{\mu}^{\prime} \theta_{\mu \nu}^{i}(s), \\
k_{\nu}^{i}(s) \equiv \partial_{\mu} \tilde{\theta}_{\mu \nu}^{i}(s) / 2 \pi=\partial_{\mu} \tilde{n}_{\mu \nu}^{i} \in \mathbb{Z},
\end{gathered}
$$

where $\partial_{\mu}^{\prime}$ is the backward derivative, and $\tilde{\theta}_{\mu \nu}$ denotes the dual tensor of $\tilde{\theta}_{\mu \nu} \equiv \frac{1}{2} \epsilon_{\mu \nu \alpha \beta} \theta_{\alpha \beta}$. Both electric and monopole currents are $\mathrm{U}(1)^{2}$ gauge invariant, according to $\mathrm{U}(1)^{2}$ gauge invariance of $\theta_{\mu \nu}^{i}(s)$. In the lattice formalism, $k_{\mu}^{i}(s)$ is located at the dual lattice $L_{\text {dual }}^{4}$ of $s^{\alpha}+1 / 2$, flowing in $\mu$ direction [15]. Hereafter, we will omit the color index $i$ as appropriate.

Abelian-projected QCD thus includes both electric current $j_{\mu}$ and monopole current $k_{\mu}$, and can be decomposed into the "photon part," which only includes $j_{\mu}$, and the "monopole part," which only includes $k_{\mu}$ approximately, as follows.

First, we consider the photon part satisfying

$$
\begin{gathered}
\theta_{\mu \nu}^{\mathrm{Ph}} \equiv \bmod _{2 \pi}\left(\partial \wedge \theta^{\mathrm{Ph}}\right)_{\mu \nu}, \\
\partial_{\mu}^{\prime} \theta_{\mu \nu}^{\mathrm{Ph}}=j_{\nu}, \quad \partial_{\mu} \tilde{\theta}_{\mu \nu}^{\mathrm{Ph}}=0 .
\end{gathered}
$$

Here, we denote by $\bmod _{2 \pi}$ the principal value in $[-\pi, \pi)$. From $\partial_{\mu} \tilde{\theta}_{\mu \nu}^{\mathrm{Ph}}=0$, one can set $\theta_{\mu \nu}^{\mathrm{Ph}}=\left(\partial \wedge \theta^{\mathrm{Ph}}\right)_{\mu \nu}$ and then $\partial_{\mu}^{\prime}\left(\partial \wedge \theta^{\mathrm{Ph}}\right)_{\mu \nu}=\partial^{2} \theta_{\nu}^{\mathrm{Ph}}-\partial_{\mu}^{\prime} \partial_{\nu} \theta_{\mu}^{\mathrm{Ph}}=j_{\nu}$. In the Landau gauge $\partial_{\mu}^{\prime} \theta_{\mu}^{\mathrm{Ph}}=0$, the photon part $\theta_{\nu}^{\mathrm{Ph}}$ can be derived from the electric current $j_{\nu}$,

$$
\partial^{2} \theta_{\nu}^{\mathrm{Ph}}=j_{\nu}, \quad \theta_{\nu}^{\mathrm{Ph}}=\frac{1}{\partial^{2}} j_{\nu} .
$$

Therefore, we here define the photon part $\theta_{\nu}^{\mathrm{Ph}}$ by

$$
\theta_{\nu}^{\mathrm{Ph}}(s) \equiv \sum_{s^{\prime}}\left\langle s\left|\frac{1}{\partial^{2}}\right| s^{\prime}\right\rangle j_{\nu}\left(s^{\prime}\right)
$$

using the inverse d'Alembertian on the lattice [15],

$$
\left\langle s\left|\frac{1}{\partial^{2}}\right| s^{\prime}\right\rangle=f\left(\left(s-s^{\prime}\right) a\right)
$$

with

$$
f(s a) \equiv-\frac{a^{2}}{4} \int_{-\frac{\pi}{a}}^{\frac{\pi}{a}} \frac{d^{4} p}{(2 \pi)^{4}} \frac{e^{-i p_{\alpha} s_{\alpha} a}}{\sum_{\mu=1}^{4} \sin ^{2}\left(p_{\mu} a / 2\right)}
$$

Here, we have explicitly written the lattice spacing $a$. Note that this function satisfies
TABLE I. The monopole density $\rho_{\mathrm{M}}$ and the electric-current density $\rho_{\mathrm{E}}$ for Abelian-projected QCD and monopole and photon parts.

\begin{tabular}{lccc}
\hline \hline Lattice & Field sector & Monopole density & Electric density \\
\hline$V=16^{3} \times 16$ & Abel & $2.95(2) \times 10^{-2}$ & $6.932(1)$ \\
$\beta=6.0$ & Monopole & $2.37(2) \times 10^{-2}$ & $0.0967(5)$ \\
& Photon & $1.39(3) \times 10^{-4}$ & $6.906(1)$ \\
$V=24^{3} \times 24$ & Abel & $2.94(1) \times 10^{-2}$ & $6.9307(7)$ \\
$\beta=6.0$ & Monopole & $2.35(1) \times 10^{-2}$ & $0.0964(4)$ \\
& Photon & $1.39(2) \times 10^{-4}$ & $6.9048(7)$ \\
$V=32^{3} \times 32$ & Abel & $1.065(5) \times 10^{-2}$ & $6.5190(5)$ \\
$\beta=6.2$ & Monopole & $0.842(5) \times 10^{-2}$ & $0.0338(1)$ \\
& Photon & $4.01(9) \times 10^{-5}$ & $6.5100(4)$ \\
$V=24^{3} \times 6$ & Abel & $1.720(9) \times 10^{-2}$ & $6.8760(8)$ \\
$\beta=6.0$ & Monopole & $1.229(8) \times 10^{-2}$ & $0.0654(3)$ \\
& Photon & $9.2(2) \times 10^{-5}$ & $6.8598(7)$ \\
\hline \hline
\end{tabular}

$$
\begin{aligned}
\partial^{2} f(s a) & \equiv \partial_{\mu} \partial_{\mu}^{\prime} f(s a) \\
& =\frac{1}{a^{2}} \sum_{\mu=1}^{4}[f(s a+\hat{\mu})+f(s a-\hat{\mu})-2 f(s a)] \\
& =\int_{-\frac{\pi}{a}}^{\frac{\pi}{a}} \frac{d^{4} p}{2 \pi)^{4}} e^{-i p_{\alpha} s_{\alpha} a},
\end{aligned}
$$

which goes to the four-dimensional delta function in the continuum limit $a \rightarrow 0$. On finite-size lattices, the momentum integral in Eq. (13) becomes a discretized sum over the momentum-space lattice.

The monopole part $\theta_{\mu}^{\mathrm{Mo}}(s)$ is defined as

$$
\theta_{\mu}^{\mathrm{Mo}}(s) \equiv \theta_{\mu}(s)-\theta_{\mu}^{\mathrm{Ph}}(s)
$$

and approximately satisfies

$$
\begin{gathered}
\theta_{\mu \nu}^{\mathrm{Mo}} \equiv \bmod _{2 \pi}\left(\partial \wedge \theta^{\mathrm{Mo}}\right)_{\mu \nu}, \\
\partial_{\mu}^{\prime} \theta_{\mu \nu}^{\mathrm{Mo}} \simeq 0, \quad \partial_{\mu} \tilde{\theta}_{\mu \nu}^{\mathrm{Mo}} \simeq k_{\nu} .
\end{gathered}
$$

In this way, in Abelian-projected QCD, the contributions from the electric current $j_{\mu}$ and the magnetic current $k_{\mu}$ can be well separated into the photon part $\theta_{\mu}^{\mathrm{Ph}}$ and the monopole part $\theta_{\mu}^{\mathrm{Mo}}$, respectively.

In Table I, we show the monopole density $\rho_{\mathrm{M}}$ and the electric-current density $\rho_{\mathrm{E}}$ defined as

$$
\begin{gathered}
\rho_{\mathrm{M}} \equiv \frac{1}{3 V} \sum_{i=1}^{3} \sum_{s, \mu}\left|k_{\mu}^{i}(s)\right|, \\
\rho_{\mathrm{E}} \equiv \frac{1}{3 V} \sum_{i=1}^{3} \sum_{s, \mu}\left|j_{\mu}^{i}(s)\right|
\end{gathered}
$$

for Abelian-projected QCD, monopole and photon parts, respectively. 
Using the monopole and the photon link variables,

$$
\begin{gathered}
u_{\mu}^{\mathrm{Mo}}(s) \equiv e^{i \theta_{\mu}^{\mathrm{Mo}}(s)} \in \mathrm{U}(1)^{2}, \\
u_{\mu}^{\mathrm{Ph}}(s) \equiv e^{i \theta_{\mu}^{\mathrm{Ph}}(s)} \in \mathrm{U}(1)^{2},
\end{gathered}
$$

monopole and photon projection are defined by the replacement of $\left\{u_{\mu}(s)\right\} \rightarrow\left\{u_{\mu}^{\mathrm{Mo}}(s)\right\},\left\{u_{\mu}^{\mathrm{Ph}}(s)\right\}$. The dominant role of the monopole part is called "monopole dominance," and monopole dominance has been observed for quark confinement in lattice QCD [13].

\section{CHIRAL CONDENSATE}

First, we study Abelian dominance and monopole dominance for the chiral condensate in the chiral limit, using the Kogut-Susskind (KS) fermion [16] for quarks in SU(3) lattice QCD.

Mathematically, the chiral condensate $\langle\bar{q} q\rangle$ in the chiral limit is directly related to the low-lying Dirac eigenvalue density $\rho(0)$ through the Banks-Casher relation [4],

$$
\langle\bar{q} q\rangle=-\lim _{m \rightarrow 0} \lim _{V \rightarrow \infty} \pi \rho(0) .
$$

The Dirac eigenvalue density $\rho(\lambda)$ is defined as

$$
\rho(\lambda) \equiv \frac{1}{V} \sum_{n}\left\langle\delta\left(\lambda-\lambda_{n}\right)\right\rangle, \quad \gamma_{\mu} D_{\mu}|n\rangle=i \lambda_{n}|n\rangle
$$

with the space-time volume $V$.
For the KS fermion, the Dirac operator $\gamma_{\mu} D_{\mu}$ becomes $\eta_{\mu} D_{\mu}$ with the staggered phase $\eta_{\mu}(s) \equiv(-1)^{s_{1}+\cdots+s_{\mu-1}}$ $(\mu \geq 2)$ with $\eta_{1}(s) \equiv 1$. The KS Dirac operator takes the explicit form of

$$
\begin{aligned}
\left(\eta_{\mu} D_{\mu}\right)_{s s^{\prime}} & =\frac{1}{2} \sum_{\mu=1}^{4} \eta_{\mu}(s)\left[U_{\mu}(s) \delta_{s+\hat{\mu}, s^{\prime}}-U_{-\mu}(s) \delta_{s-\hat{\mu}, s^{\prime}}\right] \\
& =\frac{1}{2} \sum_{\mu=1}^{4} \sum_{ \pm} \pm \eta_{\mu}(s) U_{ \pm \mu}(s) \delta_{s \pm \hat{\mu}, s^{\prime}}
\end{aligned}
$$

and hence the Dirac eigenvalue $\lambda_{n}$ is obtained from

$$
\frac{1}{2} \sum_{\mu=1}^{4} \sum_{ \pm} \pm \eta_{\mu}(s) U_{ \pm \mu}(s) \chi_{n}(s \pm \hat{\mu})=i \lambda_{n} \chi_{n}(s) .
$$

Here, the quark field $q^{\alpha}(x)$ is described by a spinless Grassmann variable $\chi(x)$, and the chiral condensate per flavor is given as $\langle\bar{q} q\rangle=\langle\bar{\chi} \chi\rangle / 4$ in the continuum limit.

For the four types of different lattices, we show in Fig. 1 the Dirac eigenvalue densities $\rho(\lambda)$ for $\mathrm{SU}(3) \mathrm{QCD}$, Abelian-projected QCD, monopole and photon sectors, extracted from lattice QCD in the MA gauge as functions of the Dirac eigenvalue $\lambda$. Figures 1(a), 1(b), and 1(c) are almost zero-temperature results in the confined phase, and

(b)
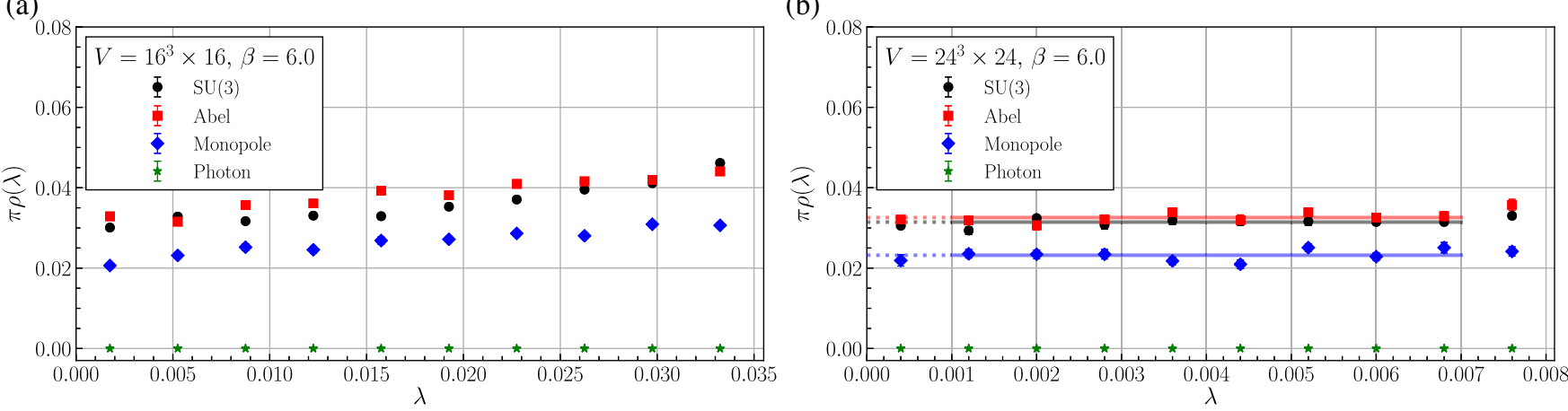

(c)

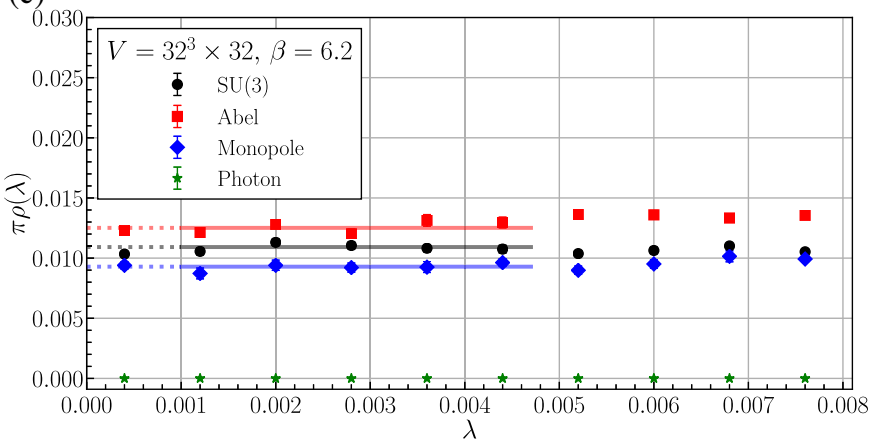

(d)

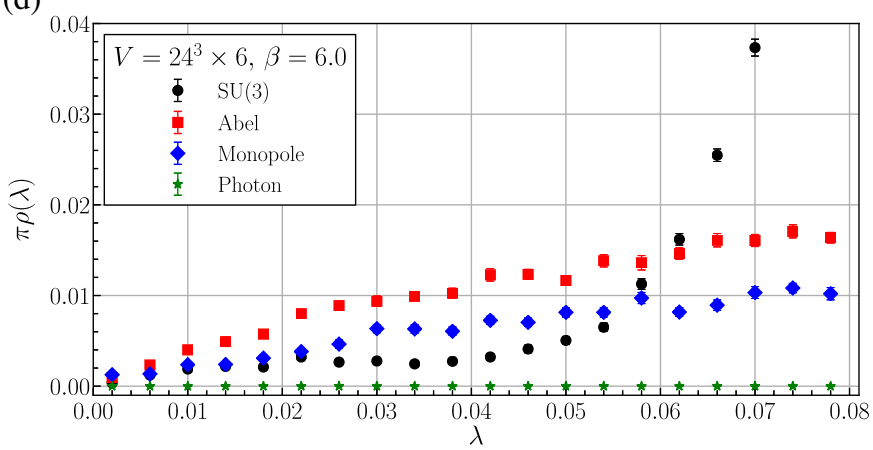

FIG. 1. The Dirac eigenvalue densities $\rho(\lambda)$ for SU(3) QCD, Abelian-projected QCD, and monopole and photon sectors, as functions of the Dirac eigenvalue $\lambda$, for the four types of different lattices: (a) $16^{3} \times 16$ at $\beta=6.0$, (b) $24^{3} \times 24$ at $\beta=6.0$, (c) $32^{3} \times 32$ at $\beta=6.2$, and (d) $24^{3} \times 6$ at $\beta=6.0$. The line is the best fit with a constant for the low-lying Dirac eigenvalue density. 
Fig. 1(d) exhibits the high-temperature deconfined phase above the critical temperature.

For all the four lattices, we find that the low-lying Dirac eigenvalue density $\rho(0)$ in Abelian-projected QCD takes approximately the same value in $\mathrm{SU}(3) \mathrm{QCD}$, which means Abelian dominance for the chiral condensate in the chiral limit. For the photon sector, we find no eigenvalues below $0.20,0.13,0.098$, and 0.27 in 10 configurations for the four different lattices of (a), (b), (c), and (d), respectively, and conclude that $\rho(0)$ in the photon sector is exactly zero. On the other hand, $\rho(0)$ in the monopole part is close to that in SU(3) QCD, which means monopole dominance for the chiral condensate in the chiral limit. Also, in the high-temperature deconfined phase of Fig. 1(d), one finds $\rho(0) \simeq 0$ for all the sectors, which physically means chiral restoration.

Next, we calculate the chiral condensate in a different way using the quark propagator. Here, we adopt the KS fermion with the bare quark mass $m$, and consider the chiral extrapolation of $m \rightarrow 0$.

For the gauge-field ensemble of $U=\left\{U_{\mu}(s)\right\}$, the Euclidean KS fermion propagator is given by the inverse matrix,

$$
\begin{aligned}
G_{U}^{i j}(x, y) & \equiv\left\langle\chi^{i}(x) \bar{\chi}^{j}(y)\right\rangle_{U}, \\
& =\left\langle x, i\left|\left(\frac{1}{\eta_{\mu} D_{\mu}[U]+m}\right)\right| y, j\right\rangle,
\end{aligned}
$$
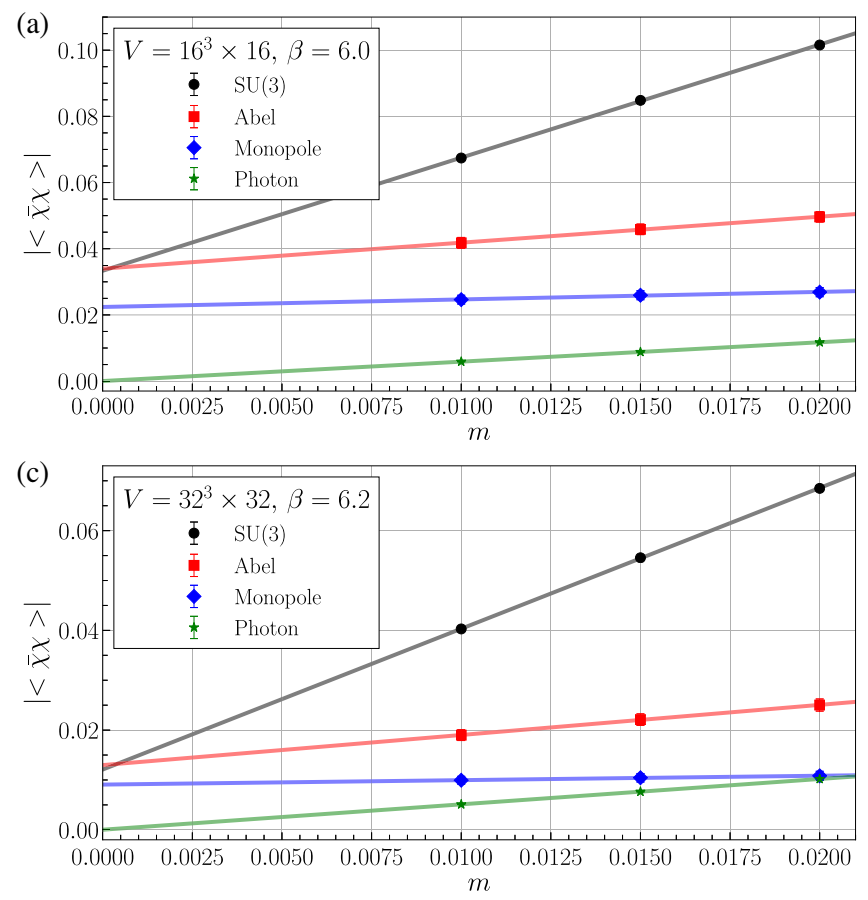

with the color index $i$ and $j$. The propagator is calculated by solving the large-scale linear equation with a point source. Using the propagator for the gauge-field ensemble $\left\{U_{\mu}(s)\right\},\left\{u_{\mu}(s)\right\},\left\{u_{\mu}^{\mathrm{Mo}}(s)\right\}$, and $\left\{u_{\mu}^{\mathrm{Ph}}(s)\right\}$, we calculate the local chiral condensate

$$
\langle\bar{\chi}(x) \chi(x)\rangle_{U}=-\operatorname{Tr} G_{U}(x, x)
$$

for SU(3) QCD, Abelian-projected QCD, and monopole and photon sectors, respectively. Here, we use 100 gauge configurations, and calculate the local chiral condensate at $2^{4}$ distant space-time points $x$ for each gauge configuration. In fact, we perform 1600 times calculations of $\langle\bar{\chi}(x) \chi(x)\rangle_{U}$ for each sector, quark mass $m$, and type of lattice. Here, we consider the net chiral condensate by subtracting the contribution from the trivial vacuum $U=1$ as

$$
\langle\bar{\chi} \chi(x)\rangle_{U} \equiv\langle\bar{\chi}(x) \chi(x)\rangle_{U}-\langle\bar{\chi} \chi\rangle_{U=1},
$$

where the subtraction term is exactly zero at the chiral limit $m=0$. We eventually take its average over the space-time $x$ and the gauge ensembles $U_{1}, U_{2}, \ldots, U_{N}$,

$$
\langle\bar{\chi} \chi\rangle \equiv \sum_{x, i}\langle\bar{\chi} \chi(x)\rangle_{U_{i}} / \sum_{x, i} 1 .
$$

For the four types of different lattices, we show in Fig. 2 the chiral condensates plotted against the bare quark mass $m$ in the lattice unit, for SU(3), Abelian, and monopole
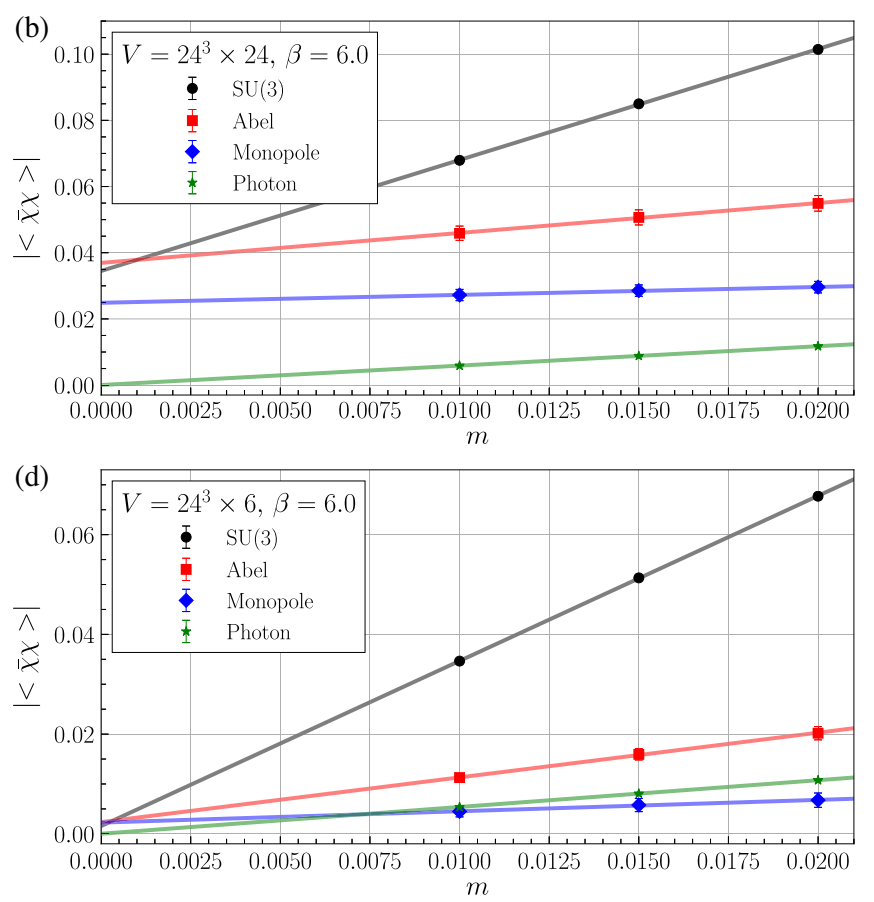

FIG. 2. The chiral condensates for SU(3) QCD, Abelian-projected QCD, and monopole and photon sectors, as functions of the bare quark mass $m$ in the lattice unit, for the four types of different lattices: (a) $16^{3} \times 16$ at $\beta=6.0$, (b) $24^{3} \times 24$ at $\beta=6.0$, (c) $32^{3} \times 32$ at $\beta=6.2$, and (d) $24^{3} \times 6$ at $\beta=6.0$. The solid line is the best fit with a linear function. 
TABLE II. The chiral condensate values in the chiral limit evaluated from the two different methods for SU(3) QCD, Abelian-projected QCD, monopole and photon sectors.

\begin{tabular}{lccr}
\hline \hline Lattice & Field sector & Banks-Casher & Propagator \\
\hline$V=24^{3} \times 24$ & SU(3) & $3.14(3) \times 10^{-2}$ & $3.45(5) \times 10^{-2}$ \\
$\beta=6.0$ & Abel & $3.26(4) \times 10^{-2}$ & $3.69(5) \times 10^{-2}$ \\
& Monopole & $2.32(5) \times 10^{-2}$ & $2.49(2) \times 10^{-2}$ \\
& Photon & $0.00(0)$ & $2.5(7) \times 10^{-5}$ \\
$V=32^{3} \times 32$ & SU(3) & $1.09(1) \times 10^{-2}$ & $1.21(3) \times 10^{-2}$ \\
$\beta=6.2$ & Abel & $1.25(2) \times 10^{-2}$ & $1.30(2) \times 10^{-2}$ \\
& Monopole & $0.93(1) \times 10^{-2}$ & $0.91(1) \times 10^{-2}$ \\
& Photon & $0.00(0)$ & $2.4(7) \times 10^{-5}$ \\
\hline \hline
\end{tabular}

and photon sectors, extracted from lattice QCD in the MA gauge.

For each sector, $m$ dependence of the chiral condensate seems to be linear in this region, and therefore we evaluate the chiral condensate in the chiral limit using the linear chiral extrapolation. Provided that the linear chiral extrapolation is valid, Abelian dominance and monopole dominance for the chiral condensate are realized in the chiral limit, whereas the photon part has almost no chiral condensate in the chiral limit.

These results are consistent with the above-mentioned conclusions using the Dirac eigenvalue density $\rho(\lambda)$ and the Banks-Casher relation. In Table II, we summarize the chiral condensate values in the chiral limit evaluated from the two different methods for SU(3), Abelian, and monopole and photon sectors.

In the presence of bare quark masses of $m=0.01-0.02$, however, there appears a significant deviation of the chiral condensates between SU(3) and Abelian sectors, which quantitatively differs from $\mathrm{SU}(2) \mathrm{QCD}$, where Abelian dominance is observed at $m=0.05-0.3$ [21]. In particular, compared with $\mathrm{SU}(3) \mathrm{QCD}$, bare-quark mass $m$ dependence of the chiral condensate is fairly reduced in Abelianprojected QCD and also in the monopole part.

As an interesting possibility, the net chiral condensate in the Abelian/monopole sector is controlled by quark-mass independent object. This might be understood if monopoles are directly responsible for chiral symmetry breaking because monopoles have no bare quark mass dependence in the quenched approximation. Then, we next examine the correlation between the chiral condensate and monopoles in more direct manner.

\section{LOCAL CORRELATION}

Second, we study the local correlation between chiral condensate and monopoles by investigating the local chiral condensate around monopoles in Abelian-projected QCD at each gauge configuration. Note that, at each lattice configuration, the monopoles topologically appear as local objects so that they might locally influence the chiral

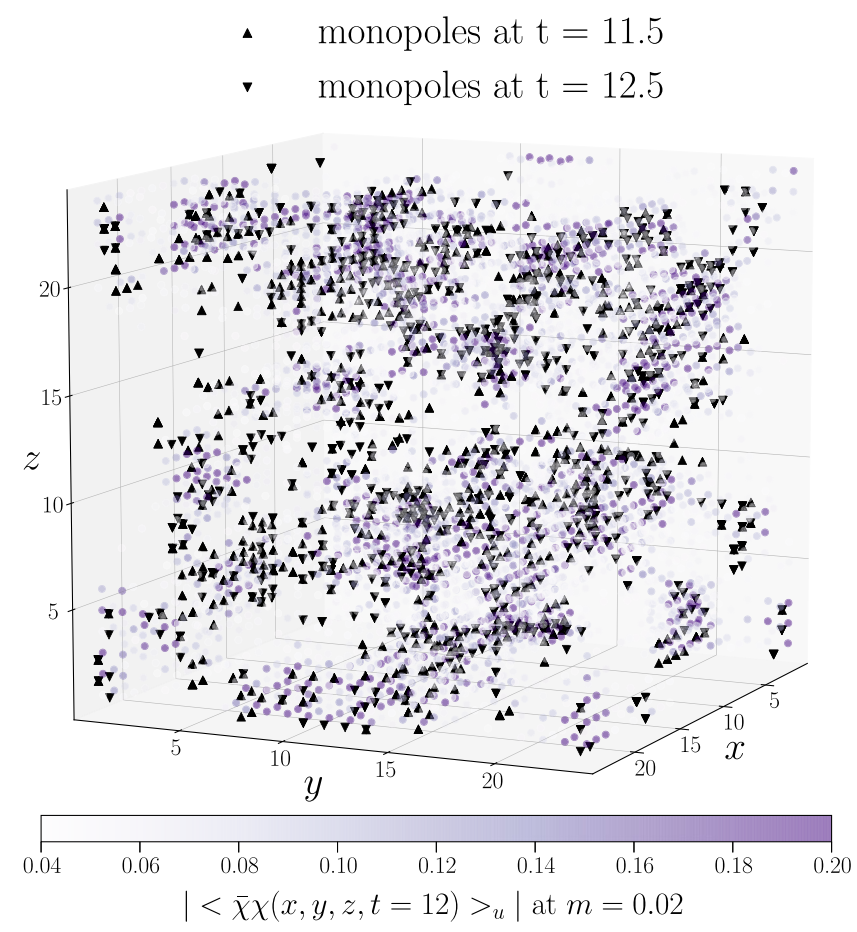

FIG. 3. The local chiral condensate at $t=12$ and monopoles at $t=11.5,12.5$ for a typical Abelian configuration of the $24^{3} \times 24$ lattice at $\beta=6.0$. The bare quark mass is taken as $m=0.02$. The value of the local chiral condensate $\left|\langle\bar{\chi} \chi(x)\rangle_{u}\right|$ is visualized with the color graduation. Monopoles at $t=11.5$ and 12.5 are plotted with upper and lower triangles, respectively.

condensate around them, although the translational invariance is recovered by the gauge ensemble average.

For the visual demonstration, we show in Fig. 3 the local chiral condensate $\langle\bar{\chi} \chi(x)\rangle_{u}$ and the monopole location at all three-dimensional space points at a time slice of $t=12$ in a typical Abelian configuration of the $24^{3} \times 24$ lattice at $\beta=6.0$. The bare quark mass is taken as $m=0.02$. Here, we show all the monopoles located at $t=11.5,12.5$ on the dual lattice $L_{\text {dual }}^{4}$ of $s^{\alpha}+1 / 2$. The value of the local chiral condensate $\left|\langle\bar{\chi} \chi(x)\rangle_{u}\right|$ is visualized with the color graduation. (The same dark color is used for $\left|\langle\bar{\chi} \chi(x)\rangle_{u}\right| \geq 0.20$, and no color is used for small $\left|\langle\bar{\chi} \chi(x)\rangle_{u}\right|<0.04$.)

It is clearly observed that the local chiral condensate in a configuration has a large fluctuation and takes quite large values in the vicinity of the monopoles.

Finally, we calculate the correlation function between the local chiral condensate $\langle\bar{\chi} \chi(x)\rangle_{u}$ and the local monopole density

$$
\rho_{\mathrm{L}}(s) \equiv \frac{1}{3 \times 2^{4}} \sum_{i=1}^{3} \sum_{s^{\prime} \in P(s)} \sum_{\mu=1}^{4}\left|k_{\mu}^{i}\left(s^{\prime}\right)\right|,
$$

where $P(s)$ denotes the dual lattices in the vicinity of $s$, i.e., $P(s)=\left\{s^{\prime} \in L_{\text {dual }}^{4}|| s^{\prime}-s \mid=1\right\}$ with the dual lattice $L_{\text {dual }}^{4}$ 

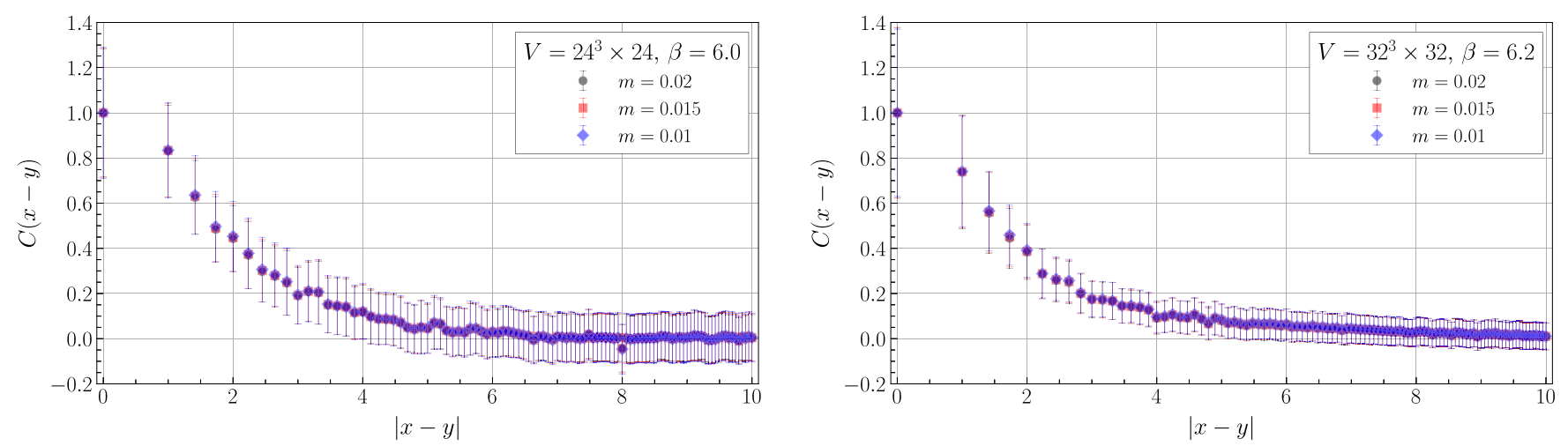

FIG. 4. The correlation function $C(x-y)$ between the local chiral condensate $\langle\bar{\chi} \chi(x)\rangle_{u}$ and the local monopole density $\rho_{\mathrm{L}}(y)$ plotted against $|x-y|$ for (left) $24^{3} \times 24$ at $\beta=6.0$ and (right) $32^{3} \times 32$ at $\beta=6.2$. The bare quark masses of $m=0.02,0.015$, and 0.01 are used in the lattice unit.

of $s^{\alpha}+1 / 2$. For this calculation, we use the lattice data of the local chiral condensate and the monopole current for 100 gauge configurations, which were used to obtain Fig. 2.

Figure 4 shows the correlation function $C(x-y)$ between the local chiral condensate $\langle\bar{\chi} \chi(x)\rangle_{u}$ and the local monopole density $\rho_{\mathrm{L}}(y)$,

$$
C(x-y) \propto\left\langle\bar{\chi} \chi(x) \rho_{\mathrm{L}}(y)\right\rangle_{u}-\langle\bar{\chi} \chi\rangle_{u}\left\langle\rho_{\mathrm{L}}\right\rangle_{u},
$$

as the function of $|x-y|$, for $24^{3} \times 24$ at $\beta=6.0$ and $32^{3} \times 32$ at $\beta=6.2$. In both lattices, the bare quark masses of $m=0.02,0.015$, and 0.01 are used, and the correlation function $C(x-y)$ is normalized to be unity at $|x-y|=0$ at each $m$. Within the error bar, the correlation function $C(x-y)$ seems to be a single-valued function of $|x-y|$, and no significant $m$ dependence of the correlation function is found in this bare quark-mass region.

It is likely that the correlation function $C(x-y)$ monotonically decreases with the distance $r \equiv|x-y|$ and almost vanishes for large $r$ such as $r \gtrsim 0.5 \mathrm{fm}$, and thus a strong correlation between the local chiral condensate and the monopole density is quantitatively clarified.

From these lattice QCD results, we conclude that there exists a direct clear local correlation between monopoles and the chiral condensate.

\section{SUMMARY AND CONCLUSION}

We have studied spontaneous chiral-symmetry breaking in SU(3) QCD in terms of the dual superconductor picture for quark confinement in the MA gauge, using lattice QCD Monte Carlo simulations with four types of different lattices. In the MA gauge, there topologically appear color-magnetic monopoles, which would be responsible to quark confinement.

First, in the confinement phase, we have found Abelian dominance and monopole dominance for the chiral condensate in the chiral limit, using the two different methods of (i) the Banks-Casher relation with the Dirac eigenvalue spectral density and (ii) finite quark-mass calculations with the quark propagator and its chiral extrapolation. We have also found that bare-quark mass dependence of the chiral condensate is fairly reduced in Abelian-projected QCD and the monopole part. In the high-temperature doconfined phase, the chiral restoration is observed also for the Abelian and the monopole sectors.

Second, we have investigated local correlation between the chiral condensate and color-magnetic monopoles, and have found that the chiral condensate takes a quite large value near the monopoles in Abelian-projected QCD.

Here, let us consider the physical origin of the correlation between chiral symmetry breaking and monopoles in terms of the magnetic catalysis. In Abelian gauge theories, chiral symmetry breaking is generally enhanced in the presence of a strong magnetic field, which is called the magnetic catalysis [25-27]. In the MA gauge, infrared QCD resembles an Abelian gauge theory with monopoles, which accompany a strong color-magnetic field around them. Therefore, as an interesting possibility, the strong magnetic field around the monopoles enhances chiral symmetry breaking also in this Abelian gauge theory.

As a future study, more detailed analysis on the local correlation would be desired to determine what is the direct trigger of the enhancement of the local chiral condensate, that is, the magnetic fields around monopoles, the presence of monopoles itself, or something else. It is also meaningful and important to investigate the effect of dynamical quarks using full QCD.

\section{ACKNOWLEDGMENTS}

H.S. is supported in part by the Grants-in-Aid for Scientific Research (Grant No. 19K03869) from the Japan Society for the Promotion of Science. Most of numerical calculations have been performed on NEC SX-ACE and OCTOPUS at Osaka University, and Yukawa-21 at YITP, Kyoto University. We have used PETSc and SLEPc to solve linear equations and eigenvalue problems for the Dirac operator, respectively [28-31]. 
[1] Y. Nambu and G. Jona-Lasinio, Dynamical model of elementary particles based on an analogy with superconductivity. I, Phys. Rev. 122, 345 (1961).

[2] K. Higashijima, Dynamical chiral-symmetry breaking, Phys. Rev. D 29, 1228 (1984).

[3] P. Zyla et al. (Particle Data Group), Review of particle physics, Prog. Theor. Exp. Phys. (2020), $083 \mathrm{C} 01$.

[4] T. Banks and A. Casher, Chiral symmetry breaking in confining theories, Nucl. Phys. B169, 103 (1980).

[5] Y. Nambu, Strings, monopoles, and gauge fields, Phys. Rev. D 10, 4262 (1974).

[6] G. 't Hooft, Gauge theories with unified weak, electromagnetic and strong interactions, in High-Energy Particle Physics (Editorice Compositori, Bologna, 1975).

[7] S. Mandelstam, Vortices and quark confinement in nonAbelian gauge theories, Phys. Rep. 23, 245 (1976).

[8] A. S. Kronfeld, M. Laursen, G. Schierholz, and U. Wiese, Monopole condensation and color confinement, Phys. Lett. B 198, 516 (1987).

[9] T. Suzuki and I. Yotsuyanagi, Possible evidence for Abelian dominance in quark confinement, Phys. Rev. D 42, 4257 (1990).

[10] K. Amemiya and H. Suganuma, Off-diagonal gluon mass generation and infrared Abelian dominance in the maximally Abelian gauge in lattice QCD, Phys. Rev. D 60, 114509 (1999).

[11] N. Sakumichi and H. Suganuma, Perfect Abelian dominance of quark confinement in SU(3) QCD, Phys. Rev. D 90, 111501 (2014).

[12] H. Ohata and H. Suganuma, Gluonic-excitation energies and Abelian dominance in SU(3) QCD, Phys. Rev. D 102, 014512 (2020).

[13] J. D. Stack, S. D. Neiman, and R. J. Wensley, String tension from monopoles in SU(2) lattice gauge theory, Phys. Rev. D 50, 3399 (1994).

[14] B. Bakker, M. Chernodub, and M. Polikarpov, Abelian Monopoles in SU(2) Lattice Gauge Theory as Physical Objects, Phys. Rev. Lett. 80, 30 (1998).

[15] H. Ichie and H. Suganuma, Monopoles and gluon fields in QCD in the maximally abelian gauge, Nucl. Phys. B574, 70 (2000).

[16] H.-J. Rothe, Lattice Gauge Theories, 4th ed. (World Scientific, Singapore, 2012).

[17] T. M. Doi, H. Suganuma, and T. Iritani, Relation between confinement and chiral symmetry breaking in temporally odd-number lattice QCD, Phys. Rev. D 90, 094505 (2014).

[18] G. 't Hooft, Topology of the gauge condition and new confinement phases in non-abelian gauge theories, Nucl. Phys. B190, 455 (1981).
[19] H. Suganuma, S. Sasaki, and H. Toki, Color confinement, quark pair creation and dynamical chiral-symmetry breaking in the dual Ginzburg-Landau theory, Nucl. Phys. B435, 207 (1995).

[20] O. Miyamura, Chiral symmetry breaking in gauge fields dominated by monopoles on SU(2) lattices, Phys. Lett. B 353, 91 (1995).

[21] R. Woloshyn, Chiral symmetry breaking in Abelianprojected SU(2) lattice gauge theory, Phys. Rev. D 51, 6411 (1995).

[22] F. X. Lee, R. Woloshyn, and H. D. Trottier, Abelian dominance of chiral symmetry breaking in lattice QCD, Phys. Rev. D 53, 1532 (1996).

[23] S. Thurner, M. Feurstein, and H. Markum, Instantons and monopoles are locally correlated with the chiral condensate, Phys. Rev. D 56, 4039 (1997).

[24] T. A. DeGrand and D. Toussaint, Topological excitations and Monte Carlo simulation of Abelian gauge theory, Phys. Rev. D 22, 2478 (1980).

[25] H. Suganuma and T. Tatsumi, On the behavior of symmetry and phase transitions in a strong electromagnetic field, Ann. Phys. (N.Y.) 208, 470 (1991).

[26] S. Klevansky, The Nambu-Jona-Lasinio model of quantum chromodynamics, Rev. Mod. Phys. 64, 649 (1992).

[27] V. Gusynin, V. Miransky, and I. Shovkovy, Dimensional reduction and catalysis of dynamical symmetry breaking by a magnetic field, Nucl. Phys. B462, 249 (1996).

[28] S. Balay, S. Abhyankar, M. F. Adams, J. Brown, P. Brune, K. Buschelman, L. Dalcin, A. Dener, V. Eijkhout, W. D. Gropp, D. Karpeyev, D. Kaushik, M. G. Knepley, D. A. May, L. C. McInnes, R. T. Mills, T. Munson, K. Rupp, P. Sanan, B. F. Smith, S. Zampini, H. Zhang, and H. Zhang, PETSc Web page, https://www.mcs.anl.gov/petsc (2019).

[29] S. Balay, S. Abhyankar, M. F. Adams, J. Brown, P. Brune, K. Buschelman, L. Dalcin, A. Dener, V. Eijkhout, W. D. Gropp, D. Karpeyev, D. Kaushik, M. G. Knepley, D. A. May, L. C. McInnes, R. T. Mills, T. Munson, K. Rupp, P. Sanan, B. F. Smith, S. Zampini, H. Zhang, and H. Zhang, PETSc Users Manual, Technical Report No. ANL-95/11 - Revision 3.14 (Argonne National Laboratory, Argonne, 2020).

[30] S. Balay, W. D. Gropp, L. C. McInnes, and B. F. Smith, Efficient management of parallelism in object-oriented numerical software libraries, in Modern Software Tools in Scientific Computing, edited by E. Arge, A. M. Bruaset, and H. P. Langtangen (Birkhäuser Press, Boston, 1997), pp. 163-202.

[31] V. Hernandez, J. E. Roman, and V. Vidal, SLEPc: A scalable and flexible toolkit for the solution of eigenvalue problems, ACM Trans. Math. Softw. 31, 351 (2005). 